\title{
Existence of Conserved Currents in the Perturbative Sine-Gordon and Massive Thirring Models
}

\author{
J. H. Lowenstein ${ }^{1 \star}$ and E. R. Speer ${ }^{2 \star \star}$ \\ 1 Department of Physics, New York University, New York, New York 10003, USA \\ 2 Department of Mathematics, Rutgers University, New Brunswick, New Jersey 08903, USA, and \\ Department of Physics, New York University, New York, New York 10003, USA
}

\begin{abstract}
The existence of infinitely many conserved currents in the quantized sine-Gordon and massive Thirring models is proved in renormalized perturbation theory.
\end{abstract}

\section{Introduction}

In this article we prove the existence (in perturbation theory) of an infinite number of conserved currents in the quantized sine Gordon model (SGM) and massive Thirring model (MTM). By a standard argument (see, e.g., [1]) these lead to conservation laws which imply [2] that the set of initial momenta in any scattering process is equal to the set of final momenta. Our method is straightforward and relatively independent of specific details of the theories; we hope it will prove applicable in other two dimensional models.

An infinite number of conserved currents in the classical SGM and MTM have previously been given explicitly [3-7]. A direct transcription of these currents into quantum operators (e.g., using Zimmermann normal products), however, would not be expected automatically to yield conserved quantum currents, due to the familiar anomalies (anisotropies) which occur in the quantized equations of motion. One anticipates, rather, the necessity of renormalizing the coefficients of the terms in the current and possibly of adding new terms. Our approach here is different; we do not use the existence of the classical currents at all, and instead construct the quantum currents directly, order by order in the coupling constant.

The currents in the quantized theories have also been studied by a number of other authors. For the SGM, the existence of the lowest order non-trivial current $j_{3}$ has been established in $[8]$ and $[1,5]$. The latter references also put forward an argument for the general case, but we believe this argument to be incorrect (see Remark 2.6 of Sect. 2). For the MTM the existence of $j_{3}$ has been established in [1, $9,10] ;[1]$ states that the methods generalize to all currents but no details are given.

* Research supported in part by National Science Foundation Grant No. PHY74-22218 A03

$\star \star$ Research supported in part by National Science Foundation Grant No. MP 574-05783 A01 
The paper is organized as follows. In Sects. 2 and 3 we construct the currents for the SGM and MTM. Section 4 is a brief review of our method, with a discussion of those aspects which might be generalized to other two-dimensional models. Relevant mathematical results on the zeros of certain polynomials are discussed in Appendix A. An interesting peripheral issue, namely, the purely kinematical implications of the conservation of a finite number of higher currents, is treated in Appendix B.

\section{Sine-Gordon Currents}

In discussing two dimensional models we use light cone coordinates $x^{ \pm}=\left(x^{0} \pm x^{1}\right) / 2, \partial_{ \pm}=\partial_{0} \pm \partial_{1}$. For the sine-Gordon model the effective Lagrangian

$$
\begin{aligned}
\mathscr{L} & =\frac{1}{2} \partial_{\mu} \phi \partial^{\mu} \phi+\tilde{m}^{2}(\beta) \beta^{-2}(\cos \beta \phi-1)=\mathscr{L}_{0}+\mathscr{L}_{I} \\
\mathscr{L}_{0} & =\frac{1}{2}\left(\partial_{\mu} \phi \partial^{\mu} \phi-m^{2} \phi^{2}\right)
\end{aligned}
$$

is quantized using the $B P H Z$ procedure; here

$$
\tilde{m}^{2}=m^{2}+\sum_{k=1}^{\infty} a_{k} \beta^{2 k}
$$

with coefficients chosen to preserve $m$ as the physical mass. For $n$ odd and positive we seek a conserved current $j_{n}$ whose zero order term in $\beta$ is the free current $j_{n}^{0}$ :

$$
\begin{aligned}
& j_{n}^{0-}=\phi_{1} \phi_{n}, \\
& j_{n}^{0+}=m^{2} \phi \phi_{n-1},
\end{aligned}
$$

where $\phi_{k} \equiv \partial_{+}^{k} \phi$. We will construct $j_{n}$ recursively, starting from (2.1).

We begin by listing the operator products to be used; their form is suggested by the classical currents $[3,4]$. Let $a=\left(a_{1}, a_{2}, \ldots, a_{2 l}\right)$ be a finite sequence of integers, with $0 \leqq a_{1} \leqq a_{2} \leqq \ldots \leqq a_{2 l}$ and $a_{2}, \ldots, a_{2 l}$ non zero. Write $l(a)=l$, $r(a)=\sum a_{i}$,

$$
C_{a}=\left\{\begin{array}{lll}
\tilde{m}^{2} \beta^{-1} N\left[\phi_{a_{2}} \ldots \phi_{a_{2 l}} \sin \beta \phi\right], & \text { if } & a_{1}=0, \\
\tilde{m}^{2} N\left[\phi_{a_{1}} \ldots \phi_{a_{2 l}} \cos \beta \phi\right), & \text { if } & a_{1}>0,
\end{array}\right.
$$

and for $a_{1}>0$ only,

$$
B_{a}=N\left[\phi_{a_{1}} \ldots \phi_{a_{21}}\right] \text {. }
$$

Here $N\left(=N_{r(a)}\right)$ is the Zimmermann normal product with canonical dimension. We say that $B_{a}$ and $C_{a}$ have length $2 l(a)$; note that $B_{a}$ and $C_{a}$ transform like the $++\ldots+$ component of a Lorentz tensor of rank $r(a)$, and that

$$
2 l(a) \leqq r(a)+1 .
$$

Note also that this list includes all monomials in + -derivatives of $\phi$, and their products by $\sin \beta \phi$ and $\cos \beta \phi$, such that the resulting operator is even under $\phi \rightarrow-\phi$. No factors of $\phi$ occur (unless the trig functions are expanded). 


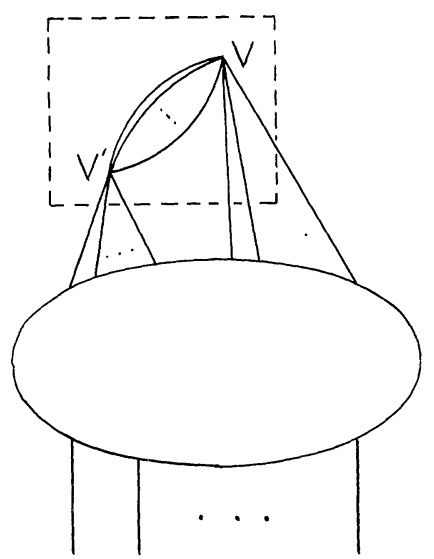

Fig. 1. Graphical structure of anomaly contribution in sine-Gordon model

Lemma 2.1. For any current of the form

$$
\begin{aligned}
& J^{-}=\sum_{r(a)=n+1} b_{a}(\beta) B_{a} \\
& J^{+}=\sum_{r(a)=n-1} c_{a}(\beta) C_{a}
\end{aligned}
$$

the divergence $\partial \cdot J=\partial_{-} J^{-}+\partial_{+} J^{+}$has the form

$$
\partial \cdot J=\sum_{r(a)=n} c_{a}^{\prime}(\beta) C_{a}+i \sum_{r(a)=n} \sum_{i=1}^{r(a)} d_{a i}(\beta) N\left[\phi_{a_{1}} \ldots \hat{\phi}_{a_{1}} \ldots \phi_{a_{2 l}(a)} \partial_{+}^{a_{1}} \frac{\delta}{\delta \phi}\right] .
$$

Here $b, c, c^{\prime}$, and $d$ are formal power series in $\beta$, and the last term is a Schwinger term, defined by

$$
\begin{aligned}
& \left\langle 0\left|T N\left[\phi_{a_{1}} \ldots \hat{\phi}_{a_{\imath}} \ldots \phi_{a_{2 l(a)}} \partial_{+}^{a_{2}} \frac{\delta}{\delta \phi}\right](x) \prod_{j=1}^{m} \phi\left(y_{j}\right)\right| 0\right\rangle \\
& =\sum_{k=1}^{m} \partial_{x+}^{a_{2}} \delta\left(x-y_{k}\right)\left\langle 0\left|\operatorname{TN}\left[\phi_{a_{1}} \ldots \hat{\phi}_{a_{\imath}} \ldots \phi_{a_{2 l(a)}}\right](x) \prod_{j \neq k} \phi\left(y_{j}\right)\right| 0\right\rangle .
\end{aligned}
$$

Such terms will be abbreviated ST in the future.

Proof. Clearly $\partial_{+} J^{+}$has the desired form, with no Schwinger terms. Using the quantized equations of motion [11] we have

$$
\begin{aligned}
\partial_{-} B_{a}= & \sum_{i=1}^{2 l(a)}\left\{-N\left[\prod_{j \neq i} \phi_{a_{j}} \partial_{+}^{a_{i}-1}\left(\tilde{m}^{2} \beta^{-1} \sin \beta \phi\right)\right]\right. \\
& \left.+i N\left[\prod_{j \neq i} \phi_{a_{j}} \partial_{+}^{a_{2}-1} \frac{\delta}{\delta \phi}\right]+m^{2} N\left[\prod_{j \neq i} \phi_{a_{j}} \partial_{+}^{a_{i}-1}(\phi-\{\phi\})\right]\right\} .
\end{aligned}
$$

The first two terms are of the form (2.4); the last is the anomalous term present only if $2 l(a) \geqq 4$, in which $\{\phi\}$ denotes that the normal product is twice oversub- 
tracted. As observed in $[1,5]$, however, the only graphs contributing to the anomaly have the form of Fig. 1, in which lines from the normal product vertex $V$, which are indexed by a set $\mathscr{X}, i \in \mathscr{X} \subset\{1, \ldots, 2 l(a)\},|\mathscr{X}| \geqq 2$, are contracted to a single interaction vertex $V^{\prime}$. The resulting operator is proportional to

$$
\tilde{m}^{2} \beta^{-2} \int d y N\left[\left(\prod_{j \notin \mathscr{X}} \phi_{a_{J}}\right)(x) \partial_{+}^{M-1}\left[\frac{\delta^{|\mathscr{X}|}}{\delta \phi(x)^{|\mathscr{X}|}} \cos \beta \phi(y)\right]\right]
$$

with $M=\sum_{j \in \mathscr{X}} a_{j}$, which is of the form (2.4).

Note in particular that if $\left(u_{k}\right)_{i}=\delta_{k i}$,

$$
\begin{aligned}
& \partial_{+} C_{a}=\sum_{k=1}^{2 l(a)} C_{a+u_{k}}+\beta^{2}(\ldots) \\
& \partial_{-} B_{a}=-\sum_{k=1}^{2 l(a)} C_{a-u_{k}}+\beta^{2}(\ldots)+\mathrm{ST}
\end{aligned}
$$

with the convention that the entries of $a \pm u_{k}$ be rearranged in increasing order.

The basic tool for constructing the currents, to be proved shortly, is

Lemma 2.2. Let $J$ be of the form (2.3), and suppose that for some $k>0$ and $2 \leqq 2 l$ $\leqq n+1,(2.4)$ becomes

$$
\partial \cdot J=\beta^{2 k}(R+\mathrm{HOT})+\mathrm{ST}
$$

with

$$
R=\sum_{l(a)=l, r(a)=n} c_{a} C_{a}
$$

$\left(c_{a} \in \mathbb{C}\right)$ and the higher order terms (HOT) either of order $\beta^{2}$ or of length greater than 2l. Then there exists a current $\mathscr{J}$ of the form (2.3) such that

$$
R=\partial \cdot \mathscr{J}+\mathrm{HOT}+\mathrm{ST} .
$$

Our main result is

Theorem 2.3. There exists a current $j_{n}$ of the form (2.3), with $\left.j_{n}\right|_{\beta=0}=j_{n}^{0}$ given by (2.1), and with $\partial \cdot j_{n}=\mathrm{ST}$.

Proof. This is an immediate consequence of Lemma 2.2. For let $J_{n}^{-}=B_{1, n}$, $J_{n}^{+}=C_{0, n-1}$; then $\left.J_{n}\right|_{\beta^{2}=0}=j_{n}^{0}$, and since $\partial \cdot J_{n}$ is of order $\beta^{2}$, we may construct $j_{n}$ by adding to $J_{n}$ currents which successively eliminate terms in $\partial \cdot j$ of increasing length, order by order in $\beta$.

Remark 2.4. If Lemma 2.2 holds for $2 l<4$, then Theorem 2.3 holds for $n=3$. For by (2.2), if we construct $j_{3}$ inductively, as described above, only terms in $\partial \cdot j$ of order 2 or 4 are encountered. 
Remark 2.5. Suppose that Theorem 3 holds for some particular $n$; we recall the derivation of the corresponding conservation law. Consider

$$
0=\int d^{2} x \partial_{x}\left\langle 0\left|T_{n}(x) \prod_{i=1}^{2 L} \phi_{i}\left(y_{i}\right)\right| 0\right\rangle^{\mathrm{con} n}
$$

(where conn denotes connected vacuum expectation values), take the Fourier transform, multiply by $\Pi\left(p_{i}^{2}-m^{2}\right)$, and restrict $p_{1} \ldots p_{k}$ to $p_{i}^{2}=m^{2}, p_{i 0}>0$; $p_{k+1} \ldots p_{2 L}$ to $p_{i}^{2}=m^{2}, p_{i 0}<0$. The integrated Schwinger terms yield

$$
F(\beta)\left(\sum_{i=1}^{2 L} p_{i+}^{n}\right){ }_{\text {out }}\left\langle p_{1} \ldots p_{k} \mid-p_{k+i} \ldots-p_{2 L}\right\rangle_{\text {in }}^{\text {conn }}
$$

where, from $(2.1), F(0)=2$. Hence

$$
\left(\sum p_{i+}^{n}\right)_{\text {out }}\left\langle p_{1} \ldots p_{k} \mid-p_{k+1} \ldots-p_{2 L}\right\rangle_{\text {in }}^{\text {conn }}=0
$$

\section{Proof of Lemma 2.2}

Case $I: 2 l \leqq 4$. For any $a$ with $r(a)=n, l(a)=l$, let $\operatorname{span}(a)=a_{2 l}-a_{1}$; since $r(a)$ is odd, $\operatorname{span}(a)>0$. We will show the existence of a current $\mathscr{J}_{a}$ with

$$
\partial \cdot \mathscr{J}_{a}=C_{a}+\sum_{\substack{l\left(a^{\prime}\right)=l, r\left(a^{\prime}\right)=n \\ \operatorname{span}\left(a^{\prime}\right)<\operatorname{span}(a)}} b_{a^{\prime}} C_{a^{\prime}}+\mathrm{HOT}+\mathrm{ST}
$$

then, using (2.11), we may successively reduce the maximum of the spans of terms in $R$ until $R$ is written as a divergence (up to HOT).

Now since $l=1$ or 2 and $r(a)=n$ is odd, $\prod_{i=1}^{2 l} \phi_{a_{\imath}}$ must have either the form

$$
\ldots\left(\phi_{a_{2 l}-1}\right)^{m} \phi_{2 l}
$$

or the form

$$
\phi_{a 1}\left(\phi_{a_{1}+1}\right)^{m^{\prime}} \ldots
$$

(or possibly both). In the first case set $\mathscr{J}_{a}^{-}=0$,

$$
\mathscr{J}_{a}^{+}=(m+1)^{-1} C_{a-u_{2 l}}
$$

and the first case does not hold, set $\mathscr{J}_{a}^{+}=0$,

$$
\mathscr{J}_{a}^{-}=\left(m^{\prime}+1\right)^{-1} B_{a+u_{1}} .
$$

(2.11) now follows immediately from (2.6). 
Case II : $2 l \geqq 6$. Consider first a general field polynomial with coefficients $b_{a} \in \mathbb{C}$ :

$$
S(x)=\sum_{l(a)=L, r(a)=m} b_{a} C_{a} .
$$

To zero order in $\beta$,

$$
\prod_{i=1}^{2 L}\left(p_{i}^{2}-m^{2}\right)\left\langle 0\left|T \tilde{S}(p) \prod_{i=1}^{2 L} \tilde{\phi}\left(p_{i}\right)\right| 0\right\rangle=i^{2 L+m} \delta\left(p+\Sigma p_{i}\right) P\left(p_{1+} \ldots p_{2 L+}\right),
$$

with

$$
P\left(p_{1+}, \ldots\right)=\sum_{\pi \in S_{2 L}} \prod_{i=1}^{2 L}\left(p_{\pi(i)+}\right)^{a_{i}} .
$$

We write $P \equiv \Lambda(S)$; the map $\Lambda$ is clearly $1-1$ and its range consists of all symmetric polynomials in $\left\{p_{i+}\right\}$, homogeneous of degree $m$, in which each monomial is independent of at most one variable. A similar map $\Lambda^{\prime}$ acts on field polynomials $\Sigma b_{a} B_{a}$, with $\Lambda^{\prime}\left(B_{a}\right)=\Lambda\left(C_{a}\right)$; since $B_{a}$ was defined only for $a_{1} \geqq 1$, polynomials in the range of $\Lambda^{\prime}$ contain a factor $\left(\Pi p_{i+}\right)$.

Now apply the reduction procedure of Remark 2.5 to (2.3) with $L=l$, keeping only terms of order at most $\beta^{2 k}$. The result is

$$
\begin{aligned}
& {\left[F(\beta)\left(\sum_{i=1}^{2 L} p_{i+}^{n}\right){ }_{\text {out }}\left\langle p_{1} \ldots p_{k} \mid-p_{k+1} \ldots p_{2 l}\right\rangle_{\text {in }}^{\text {conn }}\right]_{\text {terms of order } \leqq 2 k}} \\
& \quad+\beta^{2 k} i^{2 l+M} \delta\left(\Sigma p_{i}\right) \Lambda(R)=0
\end{aligned}
$$

for $p_{i}^{2}=m^{2}$. By Remarks 2.4 and 2.5 and Case I above, however, (2.10) holds with $n=3$ and thus ${ }_{\text {out }}\left\langle p_{1} \ldots \mid \ldots p_{2 l}\right\rangle_{\text {in }}$ vanishes unless $\sum_{1}^{2 l} p_{i+}^{3}=0$. We conclude that $\Lambda\left(p_{1+}, \ldots, p_{2 l+}\right)$ vanishes for $\Sigma p_{i+}=0$,

$$
\Sigma p_{i-}=m^{2} p_{i+}^{-1}=m^{2}\left(p_{i+}^{-1}\right) \sum_{j} \prod_{i \neq j} p_{i+}=0, \quad \text { and } \quad \sum p_{i+}^{3} \neq 0 .
$$

By Theorem 2.3 of the Appendix,

$$
\Lambda(R)\left(p_{+}\right)=i\left(\sum p_{i+}\right) Q^{+}+i\left(\sum p_{i+}^{-1}\right) Q^{-}
$$

where $Q^{+}, Q^{-}$are polynomials in $p_{i+}$ which are homogeneous of degrees $n-1$ and $n+1$ respectively; moreover, $Q^{-}$contains a factor $\Pi p_{i+}$ and hence each term in $Q^{+}$is independent of at most one $p_{i+}$ (since $\Lambda(R)$ has this property). Set $\mathscr{J}^{+}=\Lambda^{-1}\left(Q^{+}\right), \mathscr{J}^{-}=\Lambda^{-1}\left(Q^{-}\right) ;(2.6),(2.13)$ and (2.14) imply that $\Lambda(\partial \cdot \mathscr{J})=\Lambda(R)$, and since $\Lambda$ is $1-1,(2.9)$ holds.

Remark 2.6. Lemma 2.2 is not true if the condition (2.7) on $R$ is omitted. For example, equations (17) of [5] imply that any $C_{a}$ with $a_{1}=0$ (i.e., any term 
$\prod_{i=2}^{2 l} \phi_{a_{t}} \tilde{m}^{2} \beta^{-1} \sin \beta \phi$, with $\sum_{2}^{2 l} a_{i}$ odd $)$ may be written as a divergence plus Schwinger terms. Then, repeating the steps leading to (2.13), we find that $\Lambda\left(C_{a}\right)\left(p_{1+}, \ldots, p_{2 l+}\right)=0$ if $\Sigma p_{i \pm}=0, p_{i}^{2}=m^{2}$, and $\Sigma p_{i+}^{3} \neq 0$. But if $n=9$ and

$$
C_{a}=\tilde{m}^{2} \beta^{-1} \phi_{1} \phi_{2}^{4} \sin \beta \phi,
$$

then

$$
\Lambda\left(C_{a}\right)=p_{2+}\left(p_{3+} p_{4+} p_{5+} p_{6+}\right)^{2}+\text { permutations }
$$

does not vanish e.g. at $p_{1 \pm}=p_{2 \pm}=p_{3 \pm}=p_{4 \pm}=m, \quad p_{5+}=p_{6-}=(-2 \dot{+} \sqrt{3}) m$, $p_{6+}=p_{5-}=(-2-\sqrt{3}) m$. Thus the derivation of [5] is incorrect.

\section{Massive Thirring Currents}

Consider the self-interacting spinor theory with $B P H Z$ effective Lagrangian

$$
\begin{aligned}
\mathscr{L}_{\text {eff }} & =\frac{i}{2}(1+b) \bar{\psi} \gamma^{\mu} \overleftrightarrow{\partial_{\mu}} \psi-(m-a) \bar{\psi} \psi-\frac{1}{2}(g-c)\left(\bar{\psi} \gamma^{\mu} \psi\right)\left(\bar{\psi} \gamma_{\mu} \psi\right) \\
& =\mathscr{L}_{0}+\mathscr{L}_{I} \\
\mathscr{L}_{0} & =\frac{i}{2} \bar{\psi} \gamma^{\mu} \overleftrightarrow{\partial_{\mu}} \psi-m \bar{\psi} \psi
\end{aligned}
$$

where $\psi$ is a two-component spinor field, $m$ is the physical mass, $g$ is the coupling constant and $a, b$ and $c$ are finite renormalization constants which are formal power series in $g$. We adopt the representation

$$
\gamma^{0}=\gamma_{0}=\left(\begin{array}{ll}
0 & 1 \\
1 & 0
\end{array}\right), \quad \gamma^{1}=-\gamma_{1}=\left(\begin{array}{rr}
0 & 1 \\
-1 & 0
\end{array}\right)
$$

for the Dirac matrices and use positive and negative energy spinors

$$
u(p)=\left(\begin{array}{c}
p_{+}^{1 / 2} \\
p_{-}^{1 / 2}
\end{array}\right), \quad v(p)=\left(\begin{array}{c}
p_{+}^{1 / 2} \\
-p_{-}^{1 / 2}
\end{array}\right)
$$

satisfying, when $p$ is on mass shell,

$$
\begin{aligned}
(p-m) u(p) & =0=(p+m) v(p) \\
\bar{u}(p) u(p) & \equiv u^{\dagger}(p) \gamma^{0} u(p)=2 m=-\bar{v}(p) v(p) .
\end{aligned}
$$

The currents $j_{n}$ analogous to those of the sine-Gordon model have as their zeroth order terms

$$
\begin{aligned}
& j_{n}^{0-}=N\left[i \psi_{1}^{\dagger} \partial_{+}^{n} \psi_{1}+\text { h.c. }\right] \\
& j_{n}^{0+}=m N\left[\psi_{2}^{\dagger} \partial_{+}^{n-1} \psi_{1}+\text { h.c. }\right]
\end{aligned}
$$


The full current components are constructed by adding to $j_{n}^{0 \pm}$ terms of the general form

$$
\begin{aligned}
C_{a} & =N\left[i^{l} \psi_{1 a_{1}}^{\dagger} \ldots \psi_{1 a_{l}}^{\dagger} \psi_{1 a_{l+1}} \ldots \psi_{1 a_{2 l}}+\text { h.c. }\right] \\
-1 & \leqq a_{1}<a_{2}<\ldots<a_{l} \\
-1 & \leqq a_{l+1}<a_{l+2}<\ldots<a_{2 l} \\
\psi_{1 a} & =\left\{\begin{array}{ll}
\partial_{+}^{a} \psi_{1} & a \geqq 0 \\
\frac{i}{m} \psi_{2} & a
\end{array}=-1\right.
\end{aligned}
$$

where $N=N_{d(a)}$, with the canonical operator dimension $d(a)$ restricted by

$$
d(a)=r(a)+\Delta(a)
$$

and

$$
\begin{aligned}
& \Delta(a)=\text { no. of } \psi_{2} \text { and } \psi_{2}^{\dagger} \text { factors }=0,1,2, \\
& r(a)=\text { Lorentz rank }=\sum_{i=1}^{2 l}\left(a_{i}+\frac{1}{2}\right) .
\end{aligned}
$$

Note that

$$
r(a) \geqq l^{2}-\Delta(a) l
$$

i.e.

$$
l \leqq \frac{1}{2} \Delta(a)+\sqrt{r(a)+\frac{1}{4} \Delta(a)^{2}}
$$

with equality possible only for $l$ even or $\Delta(a)$ odd. The factor $i^{l}$ in the definition insures that $C_{a}$ is even under charge conjugation, $\psi_{1} \rightarrow-\psi_{1}^{\dagger}, \psi_{2} \rightarrow \psi_{2}^{\dagger}$, and further has the symmetry

$$
C_{a}=(-1)^{l} C_{\tilde{a}}
$$

where

$$
\tilde{a}=\left(a_{l+1}, \ldots, a_{2 l} ; a_{1}, \ldots, a_{l}\right) .
$$

We say that $C_{a}$ has length $2 l$.

Lemma 3.1. If

$$
\begin{aligned}
& J^{-}=\sum_{\substack{r(a)=n+1 \\
\Delta(a)=0}} c_{a}(g) C_{a} \\
& J^{+}=m^{2} \sum_{r(a)=n-1} d_{a}(g) C_{a}
\end{aligned}
$$

then

$$
\partial \cdot J=\sum_{r(a)=n} e_{a}(g) C_{a}+\mathrm{ST} .
$$


Proof. Let $\alpha$ label all distinct (up to sign and order of factors) normal products $D_{\alpha}$ of the form

$$
N\left[i^{k} \prod_{j=1}^{l} \partial_{+}^{a_{j}} \partial_{-}^{b_{j}} \psi_{c_{j}}^{\dagger} \prod_{j=l+1}^{2 l} \partial_{+}^{a_{j}} \partial_{-}^{b_{3}} \psi_{c_{j}}+\text { h.c. }\right]
$$

which are even under charge conjugation and have $\Delta(\alpha) \equiv d(\alpha)-r(\alpha) \leqq 2$. Here $\Delta(\alpha)$ counts not only the number of $\psi_{2}^{(\dagger)}$ factors, but also twice the number of $\partial_{-}$ derivatives. Each $D_{\alpha}$ which is not equal to an element of $\left\{C_{a}\right\}$ has a factor $\partial_{-} \partial_{+}^{i} \psi_{1}^{(\dagger)}, i \geqq 0$, or a factor $\partial_{+}^{j} \psi_{2}^{(\dagger)}, j \geqq 1$. If there are more than one of the latter, distinguish one arbitrarily. Applying the normal-product field Eqs. [11], one has

$$
(1+T)_{\alpha \beta} D_{\beta}=\hat{D}_{\alpha}+\tilde{D}_{\alpha}+\sum_{r(a)=r(\alpha)} X_{\alpha a} C_{a}
$$

where, if $D_{\alpha} \in\left\{C_{a}\right\}$, then

$$
\hat{D}_{\alpha}=\tilde{D}_{\alpha}=T_{\alpha \beta}=0
$$

and if $D_{\alpha} \notin\left\{C_{a}\right\}$, then

$$
\begin{aligned}
& \hat{D}_{\alpha}=D_{\alpha} \text { with }\left\{\begin{array}{l}
\partial_{-} \partial_{+}^{i} \psi_{1}^{(\dagger)} \\
\partial_{+}^{j} \psi_{2}^{(\dagger)}
\end{array}\right\} \text { replaced by }\left\{\begin{array}{l}
\partial_{+}^{i}\left(\psi_{2}^{(\dagger)}-\left\{\psi_{2}^{(\dagger)}\right\}\right) \\
\partial_{+}^{j-1}\left(\psi_{1}^{(\dagger)}-\left\{\psi_{1}^{(\dagger)}\right\}\right)
\end{array}\right\} \\
& \tilde{D}_{\alpha}=D_{\alpha} \text { with }\left\{\begin{array}{l}
\partial_{-} \partial_{+}^{i} \psi_{1}^{(\dagger)} \\
\partial_{+}^{j} \psi_{2}^{(\dagger)}
\end{array}\right\} \text { replaced by }\left\{\begin{array}{l}
\partial_{+}^{i} \delta / \delta \psi_{1}^{(\dagger)} \\
\left.\partial_{+}^{i} \delta / \delta \psi_{2}^{(\dagger)}\right\}
\end{array}\right.
\end{aligned}
$$

$T_{\alpha \beta}=0$ unless $D_{\alpha}$ has more derivatives than $D_{\beta}$ (here $\{\psi\}$ denotes one over subtraction). On the other hand, the Zimmermann identities [12] imply

$$
\hat{D}_{\alpha}=g Y_{\alpha \beta} D_{\beta}
$$

where $Y_{\alpha \beta}=0$ unless $\Delta(\alpha)=\Delta(\beta)$. Since the matrix $(1+T-g Y)$ has a perturbative inverse, Eqs. (3.6) and (3.7) can be solved for $D_{\alpha}$ as a linear combination of $C_{a}$ of the same rank, plus Schwinger terms.

Since $\partial \cdot J$ is a linear combination of $D_{\alpha}$, the Lemma is established. Note that

$$
\begin{array}{ll}
\partial_{-} C_{a}=-m^{2} \sum_{i=1}^{2 l} C_{a-u_{t}}+\mathrm{HOT}+\mathrm{ST} & (\Delta(a)=0) \\
\partial_{+} C_{a}=\sum_{i=1}^{2 l} C_{a+u_{t}}+\mathrm{HOT}+\mathrm{ST} & (\Delta(a) \leqq 2)
\end{array}
$$

where, by convention, $C_{a+u_{i}}=0$ if $a \pm u_{i}$ contains a repeated entry.

The basic tool for constructing the currents $j_{n}$ is

Lemma 3.2. Let $J$ be of the form (3.5) and suppose

$$
\partial \cdot J=g^{k}(R+\mathrm{HOT})+\mathrm{ST}
$$

with

$$
R=\sum_{\substack{r(a)=n \\ l(a)=l}} c_{a} C_{a}, \quad c_{a} \in \mathbb{C},
$$


where the higher order terms are either of order $g$ or of length greater than $2 l$. Then there exists a current $\mathscr{J}$ of the form (3.5) such that

$$
\partial \cdot \mathscr{J}=R+\mathrm{HOT}+\mathrm{ST} \text {. }
$$

Postponing the proof of the Lemma, we state our main result:

Theorem 3.3. There exists a current $j_{n}$ of the form (3.5), with $\left.j_{n}\right|_{g=0}=j_{n}^{0}$ given by (3.2), and with $\partial \cdot j_{n}=\mathrm{ST}$.

Proof. Using Lemma 3.2, we may construct $j_{n}$ by adding to $j_{n}^{0}$ currents which successively eliminate terms in $\partial \cdot j$ of increasing length, order by order in $g$.

Remark 3.4. If Lemma 3.2 holds for $2 l \leqq 4$, then Theorem 3.3 holds for $n=3$. For, by (3.4), if we construct $j_{3}$ inductively, as described above, only terms in $\partial \cdot j$ of length 2 or 4 are encountered.

Remark 3.5. The global conservation laws for scattering processes are a consequence of Theorem 3.3, integrated over all space-time. In particular

$$
\begin{aligned}
0= & \int d^{2} x\left\langle 0\left|T \partial \cdot j_{n}(x) \prod_{i=1}^{l} \psi\left(y_{i}\right) \prod_{j=1}^{l} \bar{\psi}\left(z_{j}\right)\right| 0\right\rangle \\
= & i \sum_{i=1}^{l}\left\langle 0\left|T \psi\left(y_{1}\right) \ldots \chi\left(y_{i}\right) \ldots \psi\left(y_{l}\right) \bar{\psi}\left(z_{1}\right) \ldots \bar{\psi}\left(z_{l}\right)\right| 0\right\rangle \\
& +i \sum_{j=1}^{l}\left\langle 0\left|T \psi\left(y_{1}\right) \ldots \psi\left(y_{l}\right) \bar{\psi}\left(z_{1}\right) \ldots \bar{\chi}\left(z_{j}\right) \ldots \bar{\psi}\left(z_{l}\right)\right| 0\right\rangle \\
& + \text { non-bilinear ST }
\end{aligned}
$$

where

$$
\chi_{\alpha}(x)=\delta_{\alpha 1} h_{1}(g) \partial_{+}^{n} \psi_{1}(x)+\delta_{\alpha 2}\left(h_{2}(g) \partial_{+}^{n} \psi_{2}(x)+h_{3}(g) m \partial_{+}^{n-1} \psi_{1}(x)\right) .
$$

We evaluate the Fourier transform of the right-hand side of (3.11), with all momenta near, but not on, the mass hyperbola $p_{0}^{2}=p_{1}^{2}+m^{2}$, and relabel the momenta $p_{1}, \ldots, p_{2 l}$ with $p_{i}^{0}>0$ for $i=1, \ldots, m$ and $p_{i}^{0}<0$ for $i=m+1, \ldots, 2 l$. Next, we multiply each $\tilde{\psi}\left(p_{i}\right)$ from the left by $\bar{u}\left(p_{i}\right)\left(p_{i}-m\right)$ if $p_{i}^{0}>0$ and by $\bar{v}\left(-p_{i}\right)\left(-p_{i}+m\right)$ if $p_{i}^{0}<0$, and multiply each $\tilde{\bar{\psi}}\left(p_{i}\right)$ from the right by $\left(-p_{i}-m\right) u\left(-p_{i}\right)$ if $p_{i}^{0}<0$ and by $\left(p_{i}+m\right) v(p)$ if $p_{i}^{0}>0$. Finally, we pass to the mass shells, $p_{i}^{0} \rightarrow \sqrt{p_{i 1}^{2}+m^{2}}, i=1, \ldots, m$, $p_{i}^{0} \rightarrow-\sqrt{p_{i 1}^{2}+m^{2}}, i=m+1, \ldots, 2 l$.

Making use of the identities $(i=1,2,3)$

$$
\begin{aligned}
& {\left[\bar{u}(p)(p-m) M_{i}(\not p+m)\left(p^{2}-m^{2}\right)^{-1}\right]_{p^{0}=\sqrt{p_{1}^{2}+m^{2}}}=\left.\frac{1}{2} m \bar{u}(p)\right|_{p^{0}=\sqrt{p_{1}^{2}+m^{2}}}} \\
& M_{1}=\left(\begin{array}{ll}
1 & 0 \\
0 & 0
\end{array}\right), \quad M_{2}=\left(\begin{array}{ll}
0 & 0 \\
0 & 1
\end{array}\right), \quad M_{3}=\frac{m}{p_{+}}\left(\begin{array}{ll}
0 & 0 \\
1 & 0
\end{array}\right)
\end{aligned}
$$

one verifies that the integrated bilinear ST yield

$$
\begin{aligned}
& \left(\sum_{i=1}^{3} h_{i}(g)\right)\left(\sum_{j=1}^{2 l} p_{i+}^{n}\right){ }_{\text {out }}\left\langle p_{1}, \varepsilon_{1}, \ldots, p_{m}, \varepsilon_{m} l-p_{m+1}, \varepsilon_{m+1} \ldots-p_{2 l}, \varepsilon_{2 l}\right\rangle_{\text {in }}^{\text {conn }}=0 \\
& \left(\varepsilon_{j}= \pm \text { for particle/antiparticle }\right) .
\end{aligned}
$$


Since $h_{1}(g)=-2+0(g)$ and $h_{2}(g)=0(g), h_{3}(g)=0(g)$, and the non-bilinear ST merely give $0(g)$ corrections to $h_{i}(g)$, we obtain the desired conservation law for the scattering amplitude.

\section{Proof of Lemma 3.2}

Case $I: 2 l=2$. The bilinear part of $R$ is a linear combination of $C_{a_{1} a_{2}}$ with $a_{1}+a_{2}$ even. For $a_{1}>a_{2}$, repeated use of

$$
C_{a_{1} a_{2}}=\partial_{+} C_{a_{1}-1, a_{2}}-C_{a_{1}-1, a_{2}+1}\left(+\mathrm{HOT}+\mathrm{ST} \text { if } a_{2}=-1\right)
$$

leads to

$$
C_{a_{1} a_{2}}=\partial_{+}\left[\sum_{k=1}^{1 / 2\left(a_{1}-a_{2}\right)}(-1)^{k+1} C_{a_{1}-k, a_{2}+k-1}\right]+\mathrm{HOT}+\mathrm{ST} .
$$

Case $I I: 2 l=4$. It is easy to check that any quadrilinear $C_{a}$ may be written as a linear combination of normal products

$$
N\left[\chi_{(a, b)} \chi_{(c, d)}\right] \text { and } N\left[\chi_{[a, b]} \chi_{[c, d]}\right]
$$

where

$$
\chi_{(a, b)}=\frac{1}{2}\left(\psi_{1 a}^{\dagger} \psi_{1 b}+\text { h.c. }\right), \quad \chi_{[a, b]}=\frac{1}{2}\left(i \psi_{1 a}^{\dagger} \psi_{1 b}+\text { h.c. }\right) .
$$

By repeated application, within the normal product, of

$$
\begin{gathered}
\chi_{(a, b)}=\frac{1}{2} \partial_{+}^{a-b} \chi_{(b, b)}-\sum_{1 \leqq k \leqq \frac{a-b}{2}}\left(\begin{array}{c}
a-b \\
k
\end{array}\right) \chi_{(a-k, b+k)} \\
(+\mathrm{HOT}+\mathrm{ST} \text { if } b=-1)
\end{gathered}
$$

it is clear that $\chi_{(a, b)}, a>b$, can always be written as a linear combination of $\partial_{+}^{a+b-2 c} \chi_{(c, c)}$. Similarly, $\chi_{[a, b]}, a>b$, can always be written as a linear combination of $\partial_{+}^{a+b-2 c-1} \chi_{[c+1, c]}$. Thus, it will suffice to show, for $p+q$ odd,

$$
\begin{aligned}
& A_{a b}^{p q} \equiv N\left[\partial_{+}^{p} \chi_{(a, a)} \partial_{+}^{q} \chi_{(b, b)}\right]=\partial \cdot \mathscr{J}+\mathrm{HOT}+\mathrm{ST} \\
& B_{a b}^{p q} \equiv N\left[\partial_{+}^{p} \chi_{[a+1, a]} \partial_{+}^{q} \chi_{[b+1, b]}\right]=\partial \cdot \mathscr{J}^{\prime}+\mathrm{HOT}+\mathrm{ST} .
\end{aligned}
$$

Further, without loss of generality, we may assume that $a>b$ (if $a=b$, the field product is an obvious divergence) and $p=0$ (otherwise, integrate by parts). But

$$
\begin{aligned}
A_{a b}^{0 q} & =-m^{2} \partial_{-} A_{a, b+1}^{0, q-1}-A_{a-1, b+1}^{1, q-1}+\mathrm{HOT}+\mathrm{ST} \\
& =-m^{2} \partial_{-} A_{a, b+1}^{0, q-1}-\partial_{+} A_{a-1, b+1}^{0, q-1}+A_{a-1, b+1}^{0, q}+\mathrm{HOT}+\mathrm{ST} .
\end{aligned}
$$

Iteration of (3.14) leads to

$$
A_{a b}^{0 q}=\partial \cdot \mathscr{J}^{\prime \prime}+\mathrm{HOT}+\mathrm{ST}+A_{b a}^{0 q} .
$$

But

$$
A_{b a}^{0 q}=-A_{a b}^{0 q} .
$$


Substitution of (3.16) in (3.15) yields the desired result, Eq. (3.31a). The derivation of $(3.13 \mathrm{~b})$ is strictly analogous.

Case III: $2 l \geqq 6$. The argument here parallels closely that used to prove conservation of the sine-Gordon currents. We define a one-one mapping from the set of complex linear combinations of composite fields $C_{a}$ of length $2 l$ and rank $r$ to the set of all polynomials in $p_{i+}, i=1, \ldots, 2 l$, which are (i) homogeneous of degree $r+l$, (ii) anti-symmetric under permutations of the $p_{i+}, i=1, \ldots, l$ alone, or of the $p_{i+}, i=l+1, \ldots, 2 l$, alone, and (iii) symmetric (resp. anti-symmetric) under the interchange $\left(p_{1+}, \ldots, p_{l+}\right) \leftrightarrow\left(p_{l+1+}, \ldots, p_{2 l+}\right)$ for $l$ even (resp. odd). In particular, we set

$$
\begin{aligned}
\Lambda\left(C_{a}\right)= & \sum_{\pi, \varrho}(\operatorname{sign} \pi)(\operatorname{sign} \varrho) \prod_{i=1}^{l} p_{\pi(i)+}^{a_{l}+1} \prod_{j=l+1}^{2 l} p_{\varrho(j)+}^{a_{j}+1} \\
& +(-1)^{l}\left(p_{1}, \ldots, p_{l} \leftrightarrow p_{l+1}, \ldots, p_{2 l}\right) .
\end{aligned}
$$

where the sum is over all permutations $\pi$ and $\varrho$ of $\{1, \ldots, l\}$ and $\{l+1, \ldots, 2 l\}$, respectively.

Integrating Eq. (3.9) over all space-time and applying the reduction process as in Remark 3.5, one obtains

$$
\begin{aligned}
& {\left[\left(\sum_{i=1}^{2 l} p_{i+}^{n}\right) A_{\text {out }}\left\langle p_{1}(+), \ldots, p_{l}(+) \mid-p_{l+1}(+), \ldots,-p_{2 l}(+)\right\rangle_{\text {in }}^{\text {conn }}\right]_{\text {terms of order } \leqq k}} \\
& \quad=g^{k} \delta\left(\sum_{i=1}^{2 l} p_{i}\right) \Lambda(R)
\end{aligned}
$$

where $A$ is a non-vanishing function of the momenta and $g$. Since the scattering amplitude in (3.18) vanishes for $\sum_{i=1}^{2 l} p_{i+}^{3} \neq 0$ (see Remarks 3.4 and 3.5), we conclude that $\Lambda(R)$ vanishes for all $p_{+}$such that

$$
\begin{aligned}
& \sum_{i=1}^{2 l} p_{i+}=\sum_{i=1}^{2 l} p_{i-}\left(=m^{2} \sum p_{i+}^{-1}\right)=0 \\
& \sum_{i=1}^{2 l} p_{i+}^{3} \neq 0 .
\end{aligned}
$$

By Theorem A.3,

$$
\Lambda(R)\left(p_{+}\right)=i\left(\sum_{i=1}^{2 l} p_{i+}\right) Q^{+}+i m^{2}\left(\sum_{i=1}^{2 l} p_{i+}^{-1}\right) Q^{-}
$$

where $Q^{+}, Q^{-}$are polynomials in the $p_{i+}$, homogeneous of degrees $n+l-1$ and $n+l+1$, respectively, and possessing the same (anti-)symmetry properties as $\Lambda(R)$; moreover, $Q^{-}$contains a factor $\sum_{i=1}^{2 l} p_{i+}$. Since $\Lambda$ is invertible, we may set $\mathscr{J}^{ \pm}=\Lambda^{-1}\left(Q^{ \pm}\right)$. Then, by (3.8),

$$
\Lambda(\partial \cdot \mathscr{J})=\Lambda(R) \quad(\text { HOT }+ \text { ST neglected }) .
$$

Since $\Lambda$ is one-one, (3.10) is established. This completes the proof of Lemma 3.2. 


\section{Discussion}

The proofs of the conservation laws given in Sects. 2 and 3 have essentially the same structure. Let us briefly review the common elements of the proofs, emphasizing those key ideas which might survive in an application of the method to other models.

The first step in both cases is to isolate two sets of composite-field operators: $\mathcal{O}_{C}$, the current-building terms, and $\mathcal{O}_{D}$, the terms which arise as divergences (potential obstructions). It is crucial to show that (i) $\mathcal{O}_{D}$ includes enough terms to express the divergence $\partial \cdot J$ of any current constructed from $\mathcal{O}_{C}$ (including anomalous terms arising from the field equations) and (ii) $\mathcal{O}_{C}$ includes enough terms to eliminate any obstruction in $\mathcal{O}_{D}$ arising as the divergence of a lower-order current. The verification of these complementary properties of $\mathcal{O}_{C}$ and $\mathcal{O}_{D}$ is the content of Lemmas 1 and 2 of Sects. 2 and 3. Note that in the MTM $\mathcal{O}_{C}$ and $\mathcal{O}_{D}$ essentially contain all operators of appropriate dimension and quantum numbers, while in the SGM the special form of the anomalies is used to eliminate many such terms and keep $\mathcal{O}_{C}, \mathcal{O}_{D}$ finite (for fixed $n$ ).

The proof of Lemma 2 (removal of obstructions) proceeds in three stages. First, all bilinear and quadrilinear terms in $\mathcal{O}_{D}$ are shown explicitly to be divergences (plus terms involving products of more than four fields). Second, the current $j_{3}$ is shown to be conserved in all orders. And finally, $j_{3}$ conservation is exploited to eliminate all obstructions involving products of six or more fields. (We show in Appendix $\mathrm{B}$ that the higher laws are not a purely kinematic consequence of $j_{3}$ conservation.) In both the sine-Gordon and the Massive Thirring Models, only bilinear and quadrilinear terms arise in the proof of $j_{3}$ conservation, and so the second stage is an immediate corollary of the first; it is interesting to note that this is the only role which the finiteness of the sets $\mathcal{O}_{C}$ and $\mathcal{O}_{D}$ play in the proof of all conservation laws. Conceivably there exist models in which $j_{3}$ is not restricted to involve products of length at most four, but can nevertheless be shown to be conserved; our arguments should then lead to the higher laws.

\section{Appendix A}

We wish to verify some simple properties of certain polynomials and their associated algebraic varieties. Let $\mathbb{C}[X]=\mathbb{C}\left[X_{1}, \ldots, X_{m}\right]$ (resp. $\mathbb{R}[X]$ ) denote the ring of polynomials in $X_{1} \ldots X_{m}$ with complex (resp. real) coefficients, and let $\nabla$ denote the gradient operator.

Lemma A.1. Let $f \in \mathbb{R}[X]$ be irreducible in $\mathbb{C}[X]$, and set $V=\left\{x \in \mathbb{R}^{m} \mid f(x)=0\right\}$. If for some $g \in \mathbb{C}[x]$ there exists a $y \in V$ and a real neighborhood $U$ of $y$ with $\nabla f(y) \neq 0$ and $g(x)=0$ for all $x \in V \cap U$, then $g=h f$ for some $h \in \mathbb{C}[X]$.

Proof. Let $V_{c}=\left\{z \in \mathbb{C}^{m}\{f(z)=0\}, W_{c}=\left\{z \in \mathbb{C}^{m} \mid f(z)=g(z)=0\right\}\right.$. Clearly $W_{c} \subset V_{c}$; if $W_{c}=V_{c}$, the desired result follows from Hilbert's Nullstellensatz (see, e.g., [13]). Suppose for definiteness that $\left(\nabla_{1} f\right)(y) \neq 0$; then by the implicit function theorem there exist real analytic functions $\alpha_{2}\left(x_{1}\right), \ldots, \alpha_{m}\left(x_{1}\right)$ defined near $y_{1}$ such that, in some complex neighborhood of $y, V=\left\{\left(x_{1}, \alpha\left(x_{1}\right)\right) \mid x_{1}\right.$ real $\}$ and $V_{c}=\left\{\left(z_{1}, \alpha\left(z_{1}\right)\right) \mid z_{1}\right.$ complex $\}$. But since $g(x, \alpha(x))=0$ for $x$ real, $g(z, \alpha(z))=0$ for $z$ complex, i.e., locally $W_{c}=V_{c}$. Since locally $V_{c}$ is a smooth hypersurface, $W_{c}$ and $V_{c}$ both have dimension 
$n-1$; since $V_{c}$ is irreducible, its proper subvarieties have dimension less than $n-1$, and therefore $W_{c}=V_{c}$. (See, e.g., [13, III.5.8, IV.2.3, IV.2.15].)

Now take $m=2 k$ with $k \geqq 3$. Let $\sigma_{i}(i=1, \ldots, m)$ denote the $i$ th elementary symmetric function of $X_{1}, \ldots, X_{n}$, and set $V_{1}=\left\{x \in \mathbb{R}^{m} \mid \sigma_{1}(x)=\sigma_{m-1}(x)=0\right\}$. (In applications $x_{1}, \ldots, x_{m}$ are the + components of momenta on the mass shell, $V_{1}$ the variety determined by momentum conservation.)

Lemma A.2. If for some $g \in \mathbb{C}[X]$ there is a $y \in V_{1}$ and a real neighborhood $U$ of $y$ with $\nabla \sigma_{1}$ and $\nabla \sigma_{2}$ linearly independent at $y$ and $g(x)=0$ for $x \in V_{1} \cap U$, then $g=h_{1} \sigma_{1}$ $+h_{2} \sigma_{m-1}, h_{1}, h_{2} \in \mathbb{C}[X]$.

Proof. If $h \in \mathbb{C}[X]$, the substitution $X_{m}=\sigma_{1}-\sum_{1}^{m-1} X_{i}$ allows us to write $h=\hat{h}+\tilde{h} \sigma_{1}$, where $\hat{h} \in \mathbb{C}\left[X_{1}, \ldots, X_{m-1}\right]$. The result then follows from Lemma A.1 if we can show that $\hat{\sigma}_{m-1}$ is irreducible. Let $\sigma_{i}^{\prime}, \sigma_{i}^{\prime \prime}$ denote the symmetric functions of $X_{1}, \ldots, X_{m-1}$ and $X_{1}, \ldots, X_{m-2}$, respectively. Then

$$
\hat{\sigma}_{m-1}=\sigma_{m-1}^{\prime}-\sigma_{1}^{\prime} \sigma_{m-2}^{\prime}=-\left(\sigma_{m-3}^{\prime \prime} X_{m-1}^{2}+\sigma_{1}^{\prime \prime} \sigma_{m-3}^{\prime \prime} X_{m-1}+\sigma_{1}^{\prime \prime} \sigma_{m-2}^{\prime \prime}\right)
$$

and since the discriminant $\left(\sigma_{1}^{\prime \prime} \sigma_{m-3}^{\prime \prime}\right)^{2}-4 \sigma_{1}^{\prime \prime} \sigma_{m-2}^{\prime \prime} \sigma_{m-3}^{\prime \prime}$ is not a perfect square (recall $m=2 k \geqq 6), \hat{\sigma}_{m-1}$ is irreducible.

Theorem A.3. Let $V_{3}=\left\{x \in \mathbb{R}^{m} \mid \sum x_{i}^{3}=0\right\}$. If $g \in \mathbb{C}[X]$ satisfies $g(x)=0$ for all $x \in V_{1}$, $x \notin V_{3}$, then $g=h_{1} \sigma_{1}+h_{2} \sigma_{m-1}, h_{1}, h_{2} \in \mathbb{C}[x]$.

Proof. Let $y \in \mathbb{R}^{m}$ be defined by $y_{i}=1,1 \leqq i \leqq 2 m-2, \quad y_{2 m-1}=y_{2 m}^{-1}=-(m-1)$ $+[m(m-2)]^{1 / 2}$. Then direct computation shows that $y \in V_{1}, y \notin V_{3}$, and $\nabla \sigma_{1}$ and $\nabla \sigma_{m-1}$ are linearly independent at $y$. The result follows from Lemma A.2.

\section{Appendix B}

We wish to verify that the cubic conservation law arising from $j_{3}$ does not, on purely kinematic grounds, imply higher conservation laws and the equality of the sets of incoming and outgoing momenta. We actually prove a more general result. Consider a process in which $k$ particles, momenta $p_{1}, \ldots, p_{k}$, scatter into $k^{\prime}$ particles, momenta $p_{1}^{\prime}, \ldots, p_{k^{\prime}}^{\prime}\left(k+k^{\prime}=m\right)$, subject to the conservation laws arising from the $K$ currents $j_{1}, j_{3}, \ldots, j_{2 K-1}$ and their images under parity transformation. If we set $x_{i}=p_{i+}, 1 \leqq i \leqq k$, and $x_{k+i}=-p_{i+}^{\prime}, 1 \leqq i \leqq k^{\prime}$, and use the mass shell condition, the conservation laws become

$$
F_{i}(x) \equiv \sum_{i=1}^{m} x_{i}^{j}=0, \quad j= \pm 1, \ldots, \pm(2 K-1) ;
$$

conversely, any $x$ (with non-vanishing components) satisfying (B.1) gives such a process, with $k=\left|\left\{i \mid x_{i}>0\right\}\right|, k^{\prime}=\left|\left\{i \mid x_{i}<0\right\}\right|$.

Lemma B.1. If $m>4 K$ and $k \geqq 2 K, k^{\prime} \geqq 2 K, k+k^{\prime}=m$, there exist solutions of (B.1) such that (i) $k=\left|\left\{i \mid x_{i}>0\right\}\right|, k^{\prime}=\left\{\left\{i \mid x_{i}<0\right\} \mid\right.$, and (ii) the next conservation law

$$
F_{2 K+1}(x)=0
$$

does not hold. 
Remark. Since we can take $k=2 K, k^{\prime} \neq k$, (i) suffices to conclude that $j_{1}, \ldots, j_{2 K-1}$ conservation does not imply equality of sets of in and out momenta. It can be shown, however, that this equality does hold if $m \leqq 4 K, m$ even.

Proof. Let $a_{1}, \ldots, a_{K}$ be distinct positive reals. For $p \geqq 2 K$ consider the equations

$$
\sum_{i=1}^{p} y_{i}^{j}=0, \quad j=1,3, \ldots, 2 K-1 ;
$$

their Jacobian with respect to $y_{1}, \ldots, y_{K}$,

$$
\left|\begin{array}{ccc}
1 & \cdots & 1 \\
3 y_{1}^{2} & & 3 y_{K}^{2} \\
\vdots & & \vdots \\
(2 K-1) y_{1}^{2 K-2} & & (2 K-1) y_{K}^{2 K-2}
\end{array}\right|=\prod_{j=1}^{K}(2 j-1) \prod_{1 \leqq i<j \leqq K}\left(y_{i}^{2}-y_{j}^{2}\right)
$$

does not vanish at $y=\left(a_{1}, \ldots, a_{K},-a_{1}, \ldots,-a_{K}, 0, \ldots, 0\right)$ so by the implicit function theorem there is a nearby solution $y=y^{(p)}$ with $y_{i}^{(p)}>0, i>2 K$. Now for $u \in \mathbb{R}^{k}$, $v \in \mathbb{R}^{k^{\prime}}, \lambda \in \mathbb{R}$ with non-vanishing coefficients, define

$$
\begin{aligned}
& x_{i}(u, v, \lambda)= \begin{cases}u_{i}, & 1 \leqq i \leqq k, \\
-\lambda / v_{i-k}, & k<i \leqq m ;\end{cases} \\
& \tilde{x}_{i}(u, v, \lambda)=\lambda / x_{i}(u, v, \lambda) .
\end{aligned}
$$

Then (B.1) becomes

$$
F_{j}(x(u, v, \lambda))=F_{j}(\tilde{x}(u, v, \lambda))=0, \quad j=1,3, \ldots, 2 K-1 .
$$

But $u=y^{(k)}, v=y^{\left(k^{\prime}\right)}, \lambda=0$ solves (B.4) and the Jacobian with respect to $u_{1}, \ldots, u_{K}$, $v_{1}, \ldots, v_{K}$ is non-zero there, so again there are nearby solutions. In fact, fixing $\lambda=\lambda_{0}>0$, we get a family of solutions parametrized by $u_{i}$ and $v_{i}, i>K$, any one of which gives a solution of (B.1) satisfying (i) of the Lemma. Further, (B.2) cannot hold at all these points, for the Jacobian of the $(2 K+1)$ Eqs. (B.2), (B.4) with respect to $u_{1}, \ldots, u_{K}, v_{1}, \ldots, v_{K}$, and $u_{k}$ (if $k>2 K$, otherwise $v_{k^{\prime}}$ ) is non-vanishing [as in (B.3)], and therefore, within our set of solutions, (B.2) determines $u_{k}$ in terms of $u_{i}, K<i<k$, and $v_{i}, K<i$.

Acknowledgements. We would like to thank H.-J. Thun for informative discussions. One of us (E.R.S.) would like to thank the New York University Department of Physics for hospitality while this work was done.

\section{References}

1. Nissimov, E. R.: Bulg. J. Phys. 4, 113 (1977)

2. Fadeev,L.D.: Institute for Advanced Study, Preprint (1975)

3. Krushkal, M.D., Wiley,D.: AMS Summer Seminar on Nonlinear Wave Motion. Newell, A.C., (ed.). New York: Potsdam 1972

4. Kulish,P.P., Nissimov, E. R. : JETP Lett. 24, 220 (1976)

5. Kulish,P.P., Nissimov, E. R.: Theor. Math. Phys. 29, 992 (1976)

6. Berg, B., Karowski, M., Thun,H.-J.: Phys. Lett. 64B, 286 (1976)

7. Flume, R., Mitter,P.K., Papanicolaou, N. : Phys. Lett. 64B, 289 (1976)

8. Flume, R.: Phys. Lett. 62B, 93 (1976) and 68B, 487 (1977) 
9. Berg, B., Karowski,M., Thun,H.-J.: Nuovo Cimento 38A, 11 (1977)

10. Flume, R., Meyer,S. : Nuovo Cimento Lett. 18, 238 (1977)

11. Gomes, M., Lowenstein,J.H.: Nucl. Phys. B45, 252 (1972)

12. Zimmermann, W.: Ann. Phys. 77, 536 (1973)

13. Kendig, K.: Elementary algebraic geometry. Berlin, Heidelberg, New York: Springer 1977

Communicated by R. Stora

Received June 21, 1978 